\title{
Hyperbolic Tessellation and Colorings of Trees
}

\author{
Dong Han Kim ${ }^{1}$ and Seonhee $\mathrm{Lim}^{2}$ \\ ${ }^{1}$ Department of Mathematics Education, Dongguk University-Seoul, Seoul 100-715, Republic of Korea \\ ${ }^{2}$ Department of Mathematical Sciences, Seoul National University, Seoul 151-747, Republic of Korea
}

Correspondence should be addressed to Seonhee Lim; slim@snu.ac.kr

Received 22 February 2013; Accepted 3 May 2013

Academic Editor: Baodong Zheng

Copyright (C) 2013 D. H. Kim and S. Lim. This is an open access article distributed under the Creative Commons Attribution License, which permits unrestricted use, distribution, and reproduction in any medium, provided the original work is properly cited.

\begin{abstract}
We study colorings of a tree induced from isometries of the hyperbolic plane given an ideal tessellation. We show that, for a given tessellation of the hyperbolic plane by ideal polygons, a coloring can be associated with any element of Isom $\left(\mathbb{U}^{2}\right)$, and the element is a commensurator of $\Gamma$ if and only if its associated coloring is periodic, generalizing a result of Hedlund and Morse.
\end{abstract}

\section{Introduction}

Let $T$ be a locally finite tree, $V T$ its vertex set, and $E T$ the set of oriented edges of $T$. Let $\mathscr{A}$ be a countable set which will be called the alphabet. Let $\phi$ be a coloring of $T$, that is, a map $\phi: V T \rightarrow \mathscr{A}$. Let $\operatorname{Aut}(T)$ be the automorphism group of $T$. A periodic coloring is a coloring which is $\Gamma$-invariant for some cocompact subgroup $\Gamma \subset \operatorname{Aut}(T)$.

In this paper, we study colorings of regular trees induced from some tessellations of the hyperbolic plane.

There is a well-known family of sequences coming from rotations of circle as follows. Consider the tiling of the real line $\mathbb{R}$ by unit length intervals $\{[n, n+1): n \in \mathbb{Z}\}$ and a map $t \mapsto a t+b$ from $\mathbb{R}$ to itself. There exists an integer $j$ such that each interval $[n, n+1)$ is partitioned into $j$ or $j+1$ subintervals of the form $\{[a n+b, a(n+1)+b): n \in \mathbb{Z}\} \cap\{[n, n+1): n \in \mathbb{Z}\}$. Consider the sequence $\left(u_{n}\right)_{n \in \mathbb{N}}$ with $u_{n} \in \mathscr{A}=\{j, j+1\}$, which is given by the number of such subintervals of $[n, n+1)$. It is well known that this two-sided sequence $u_{n}$ is periodic if only if $a$ is rational [1].

As a generalization, we associate a coloring $\phi_{g}$ of a $k$ regular tree $(k \geq 3)$ for any isometry $g$ of the hyperbolic plane, given a specific hyperbolic tessellation $\mathscr{D}$ generated by a discrete subgroup $\Gamma$ of the group of isometries on the hyperbolic plane $\mathbb{U}^{2}$. Suppose that each vertex of elements of $\mathscr{D}$ lies on the boundary of the hyperbolic plane so that the dual graph of $\mathscr{D}$ is a tree.

For such a tessellation $\mathscr{D}$, we show that the coloring $\phi_{g}$ is periodic if and only if $g$ is a commensurator of $\Gamma$ in $\operatorname{Isom}\left(\mathbb{H}^{2}\right)$.
Recall that an element $g \in \operatorname{Isom}\left(\mathbb{M}^{2}\right)$ is called a commensurator of $\Gamma$ if and only if $g \Gamma g^{-1} \cap \Gamma$ is a subgroup of $\Gamma$ and of $g \Gamma g^{-1}$ of finite index. Let us denote the group of commensurators of $\Gamma$ by $\operatorname{Comm}(\Gamma)$. Commensurator subgroup $\operatorname{Comm}(\Gamma)$ plays an important role in the study of rigidity of locally symmetric spaces and more generally in geometric group theory ([2-4]).

This is a result analogous to the rotation case in the sense that the group of commensurators of $\mathrm{SL}_{2}(\mathbb{Z})$ is a group containing $\mathrm{SL}_{2}(\mathbb{Q})$ with finite index [5].

After showing the main theorem (Theorem 3), we show that our construction is an analogue of sequences induced from a rotation of circle only when the multiplicative constant $a$ of $t \mapsto a t+b$ is rational.

We show that, in the case of an isometry of $\mathbb{U}^{2}$ which is not a commensurator, we obtain colorings of unbounded alphabet, in contrast with the motivating example where irrational rotations correspond to Sturmian sequences, which are in particular sequences with a finite alphabet (see Section 3 for details).

We then explain in a heuristic way how to obtain eventually periodic colorings and colorings of "low complexity" by disregarding some information of the induced colorings.

\section{Periodic Tree Colorings from Hyperbolic Tessellations}

We first reformulate the classical example of two-sided sequences mentioned in Section 1. Consider the tessellation $\mathscr{D}$ of the hyperbolic plane (upper-half plane) $\mathbb{U}^{2}$ given by the 
group $\Gamma_{r}$ generated by the reflections about the lines $x=0$ and $x=1$. More precisely, elements of $\mathscr{D}$ are of the form $\{z \in \mathbb{C}: n \leq \operatorname{Re}(z)<n+1\}$. Then $\Gamma_{r}=\langle z \mapsto-\bar{z}, z \mapsto 2-\bar{z}\rangle$ is isomorphic to the infinite dihedral group, and its dual graph $T$ is a 2-regular tree. Let $g=\left(\begin{array}{ll}a & b \\ c & d\end{array}\right) \in \mathrm{PSL}_{2}(\mathbb{R})$, which sends $z \in \mathbb{H}^{2}$ to $(a z+b) /(c z+d)$. Then it is not difficult to check that $g$ is a commensurator of $\Gamma_{r}$ if and only if $c=0$, and $a / d$ is rational.

For each vertex $x \in V T$, denote by $D_{x} \in \mathscr{D}$ the element of $\mathscr{D}$ dual to $x$. Let $\phi_{g}^{\#}$ be the coloring given by

$$
\phi_{g}^{\#}(x)=\#\left\{g D: g D \cap D_{x} \neq \emptyset, D \in \mathscr{D}\right\} .
$$

Then $\phi_{g}^{\#}$ is periodic if $a / d$ is rational, $c=0$, and Sturmian if $a / d$ is irrational, $c=0$ (e.g., [1], [9, Chapter 6]).

Let us generalize the above construction. Let us fix an ideal polygon $D$ in the hyperbolic plane $\mathbb{H}^{2}$. Consider the group $\Gamma_{r}$ generated by the reflections in the edges of $D$, which is a discrete subgroup of finite covolume in the isometry group of $\mathbb{M}^{2}$. By Poincaré's theorem on fundamental polygons, there exists a tessellation $\mathscr{D}$ of $\mathbb{H}^{2}$ by the images of $D$ by the elements of $\Gamma_{r}$.

Let $T$ be the dual graph of the tessellation $\mathscr{D}$, which is a tree since $D$ is an ideal polygon. The tree $T$ is the Cayley graph of the group $\Gamma_{r}$.

More generally, we will also consider the case when $\Gamma_{r}$ is generated by the reflections in the edges of a generalized ideal polygon, by which we mean a polygon in $\mathbb{T}^{2} \cup \partial \mathbb{H}^{2}$ such that all vertices are on the boundary $\partial \mathbb{U}^{2}$. Note that such a generalized ideal polygon may have infinite volume.

For any given $g \in \operatorname{Isom}\left(\mathbb{T}^{2}\right)$, we associate a coloring to $g$ as follows. Consider $g \mathscr{D}=\{g D: D \in \mathscr{D}\}$. For any vertex $x$ of the dual graph $T$, the polygon $D_{x}$ dual to $x$ is a union of subsets of the form $D_{x} \cap g D^{\prime}$, for $D^{\prime} \in \mathscr{D}$, with mutually disjoint interiors. We call by the partition of $D$ by $\mathscr{D} \vee g \mathscr{D}$ the collection $\left\{D \cap g D^{\prime}: D^{\prime} \in \mathscr{D}\right\}$ just described (disregarding intersections on the boundary).

Definition 1. Let $\mathscr{A}$ be the set of equivalence classes of partitions of elements of $\mathscr{D}$, where two partitions of $D$ and $D^{\prime}$, respectively, are equivalent if there exists an isometry from $D$ to $D^{\prime}$ which sends elements of the partition of $D$ bijectively to elements of the partition of $D^{\prime}$. The coloring $\phi_{g}$ associated with $g$ is the map $\phi_{g}: V T \rightarrow \mathscr{A}$ sending $x$ to the class of the partition of $D_{x}$ by $\mathscr{D} \vee g \mathscr{D}$.

Let $\Gamma_{\mathscr{D}} \subset \operatorname{Isom}\left(\mathbb{M}^{2}\right)$ be the set of isometries leaving $\mathscr{D}$ invariant. Since every $D \in \mathscr{D}$ is a generalized ideal polygon with finitely many sides, $\Gamma_{r}$ is a finite index subgroup of $\Gamma_{\mathscr{D}}$. Thus $g \in \operatorname{Isom}\left(\mathbb{M}^{2}\right)$ is a commensurator of $\Gamma_{r}$ if and only if $g$ is a commensurator of $\Gamma_{\mathscr{D}}$.

Lemma 2. For each $x, y \in V T$, one has $\phi_{g}(x)=\phi_{g}(y)$ if and only if there exists $\gamma \in \Gamma_{\mathscr{D}} \cap g \Gamma_{\mathscr{D}} g^{-1} \subset \operatorname{Isom}\left(\mathbb{H}^{2}\right)$ such that $\gamma D_{x}=D_{y}$, where $D_{x}, D_{y}$ are the elements of $\mathscr{D}$ associated with $x, y \in V T$.
Proof. Suppose that $\gamma D_{x}=D_{y}$ for some $\gamma \in \Gamma_{\mathscr{D}} \cap g \Gamma_{\mathscr{D}} g^{-1}$. Let the partition of $D_{x}$ by $\mathscr{D} \vee g \mathscr{D}$ be

$$
D_{x}=\bigcup_{i \in I}\left(D_{x} \cap g E_{i}\right) \text {, }
$$

where $\left\{E_{i}\right\}_{i \in I} \subset \mathscr{D}$. Then

$$
\gamma D_{x}=\bigcup_{i \in I}\left(\gamma D_{x} \cap \gamma g E_{i}\right)
$$

Since $\gamma \in \Gamma_{\mathscr{D}} \cap g \Gamma_{\mathscr{D}} g^{-1}$, we have $\gamma=g \gamma^{\prime} g^{-1}$ for some $\gamma^{\prime} \in \Gamma_{\mathscr{D}}$. Thus

$$
\gamma D_{x}=\bigcup_{i \in I}\left(\gamma D_{x} \cap g \gamma^{\prime} E_{i}\right)
$$

Since $g \gamma^{\prime} E_{i}$ are all elements of $g \mathscr{D}$, the above partition is a partition of $\gamma D_{x}$ by $\mathscr{D} \vee g \mathscr{D}$. Therefore, the colorings $\phi_{g}$ on $D_{x}$ and $D_{y}=\gamma D_{x}$ are the same.

Conversely, any isometry from $D_{x}$ to $D_{y}$ extends to an isometry of $\mathbb{H}^{2}$ leaving $\mathscr{D}$ invariant. Thus if $\phi_{g}(x)=\phi_{g}(y)$, then there exists $\gamma \in \Gamma_{\mathscr{D}}$ such that $\gamma D_{x}=D_{y}$ which sends elements of the partition of $D_{x}$ by $\mathscr{D} \vee \mathscr{g} \mathscr{D}$ bijectively to those of $D_{y}$. Let us denote the partitions of $D_{x}$ and $D_{y}=\gamma D_{x}$ by $\mathscr{D} \vee g \mathscr{D}$ by

$$
\begin{aligned}
& D_{x}=\bigcup_{i \in I}\left(D_{x} \cap g E_{i}\right), \\
& D_{y}=\gamma D_{x}=\bigcup_{j \in J}\left(\gamma D_{x} \cap g F_{j}\right),
\end{aligned}
$$

for some $\left\{E_{i}\right\}_{i \in I},\left\{F_{j}\right\}_{j \in J} \subset \mathscr{D}$. Since $D_{x}$ and $D_{y}$ have the same coloring, the above partition is equal to

$$
\gamma D_{x}=\bigcup_{i \in I}\left(\gamma D_{x} \cap \gamma g E_{i}\right) .
$$

Thus $I=J$, and by rearranging $F_{j}$ if necessary, we have $D_{y} \cap g F_{i}=D_{y} \cap \gamma g E_{i}$ for each $i$. Since $\mathscr{D}$ is a tessellation by ideal polygons, this implies that $g F_{i}=\gamma g E_{i}$. As $F_{i}$ and $E_{i}$ are elements of $\mathscr{D}$, there exists $\gamma_{i} \in \Gamma_{\mathscr{D}}$ such that $F_{i}=\gamma_{i} E_{i}$. Thus $g \gamma_{i}(\gamma g)^{-1}$ stabilizes $E_{i}$; thus it is an element of $\Gamma_{\mathscr{D}}$, say $\gamma^{\prime}$. We conclude that $\gamma^{\prime} \gamma=g \gamma_{i} g^{-1} \in g \Gamma_{\mathscr{D}} g^{-1}$ satisfies the statement of the lemma.

Now let us formulate our theorem.

Theorem 3. Let $\Gamma_{r}$ be a group generated by the reflections in the edges of a generalized ideal polygon. An isometry $g \in \operatorname{Isom}\left(\mathbb{H}^{2}\right)$ is a commensurator of $\Gamma_{r}$ if and only if its associated coloring $\phi_{g}$ is periodic.

Proof. As we mentioned earlier, $g \in \operatorname{Comm}\left(\Gamma_{r}\right)$ if and only if $g \in \operatorname{Comm}\left(\Gamma_{\mathscr{D}}\right)$. Suppose that $\Gamma^{\prime}=g \Gamma_{\mathscr{D}} g^{-1} \cap \Gamma_{\mathscr{D}}$ is a finiteindexed subgroup of $\Gamma_{\mathscr{D}}$. By Lemma 2 , we know that $\phi_{g}$ is $\Gamma^{\prime}$-invariant. Since $\Gamma_{\mathscr{D}}$ is cocompact in $\operatorname{Aut}(T), \Gamma^{\prime}$ is also a cocompact discrete subgroup of $\operatorname{Aut}(T)$. Thus $\phi_{g}$ is periodic.

Conversely, suppose that $\phi_{g}$ is periodic. Let $\Gamma$ be a cocompact subgroup of $\operatorname{Aut}(T)$ preserving $\phi_{g}$. For any $x \in V T$ and $\gamma \in \Gamma$ we have $\phi_{g}(x)=\phi_{g}(\gamma x)$; thus, by Lemma 2 , there exists $\gamma^{\prime} \in \Gamma^{\prime}=\Gamma_{\mathscr{D}} \cap g \Gamma_{\mathscr{D}} g^{-1}$ such that $\gamma^{\prime}\left(D_{x}\right)=D_{\gamma x}$. Letting 


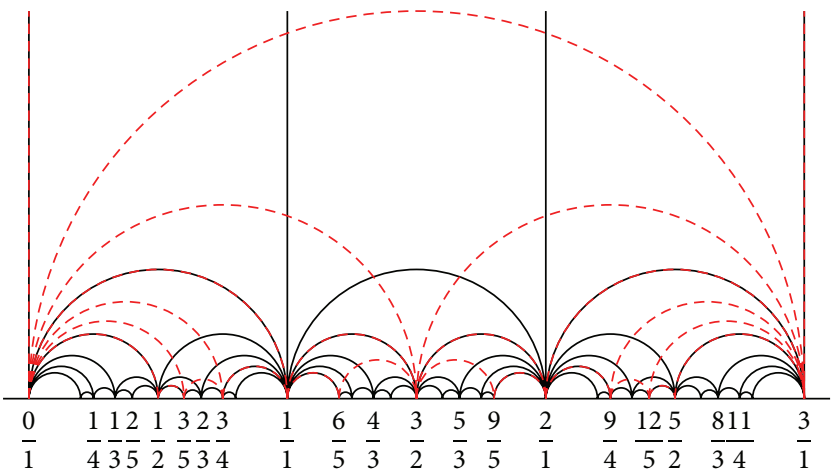

(a) $z \mapsto 3 z$

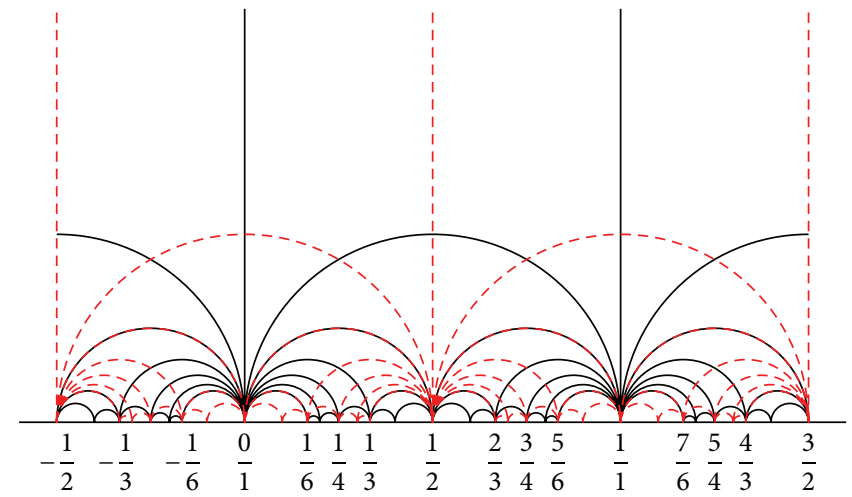

(b) $z \mapsto z+(1 / 2)$

FIGURE 1: Examples of isometry $g$ associated with periodic colorings.

$\Gamma^{\prime \prime}=\left\{\gamma^{\prime} \in \Gamma^{\prime}: \gamma \in \Gamma\right\}$, it follows that $\Gamma^{\prime \prime} \backslash T \simeq \Gamma^{\prime} \backslash T$ is finite since $\Gamma \backslash T$ is finite. Since $\Gamma_{r}$ is a finite index subgroup of $\Gamma_{\mathscr{D}}$ and $T$ is the Cayley graph of $\Gamma_{r}, \Gamma^{\prime} \subset \Gamma_{\mathscr{D}}$ is of finite index. Therefore $g$ is a commensurator of $\Gamma_{\mathscr{D}}$, thus a commensurator of $\Gamma_{r}$.

Note that the coloring $\phi_{g}^{\#}$ on a 2-regular tree in (1) is periodic if $\phi_{g}$ is periodic.

Now let us provide some examples of isometries of the hyperbolic plane giving periodic colorings. A periodic coloring which is $\Gamma$-invariant for some $\Gamma \subset \operatorname{Isom}\left(\mathbb{M}^{2}\right)$ will be expressed on the quotient, denoted by $\Gamma \backslash \backslash T$, which is either a graph (if there is no torsion element) or a graph of groups (if there are some torsion elements, we attach the stabilizers of vertices and edges on the quotient graph). In fact, we will express a coloring on the edge-indexed graph of the quotient graph of groups $\Gamma \backslash \backslash T$, as we only need the edge-indexed graph of a graph of groups to recover $T$ from a graph of groups.

Recall that the edge-indexed graph of a graph of groups is a graph with an index on each oriented edge, where the graph is given by the quotient graph $\Gamma \backslash T$ and the index $i(e)$ of the oriented edge $e$ is given by the index of the edge group $G_{e}$ in the vertex group $G_{\partial_{0}(e)}$ of the initial vertex $\partial_{0}(e)$ of $e$. (For details on graph of groups and the edge-indexed graph of a graph of groups, see [6-8].)

Example 4. Consider the Farey tessellation $\mathscr{D}$ of the hyperbolic plane, which is the tessellation with $D$ the ideal triangle of vertices $\infty, 0$, and 1 . Then $\Gamma_{r}=\langle-z, z /(2 z-1), 2-z\rangle$, and the dual graph of $\Gamma_{r}$ is a 3-regular tree $T$. Note that $\Gamma_{r} \cap \mathrm{PSL}_{2}(\mathbb{Z})$ is a subgroup of $\Gamma_{r}$ of index $2 . \Gamma_{r}$ is commensurable to $\mathrm{PSL}_{2}(\mathbb{Z})$ as $\Gamma_{r} \cap \mathrm{PSL}_{2}(\mathbb{Z})$ is a subgroup of $\mathrm{PSL}_{2}(\mathbb{Z})$ of index six since $\{z, 1 /(1-z),(z+1) / z\}$ is the stabilizer subgroup of $D$ in $\mathrm{PSL}_{2}(\mathbb{Z})$.

A hyperbolic element $g_{1}: z \mapsto 3 z$ and a parabolic element $g_{2}: z \mapsto z+(1 / 2)$ is considered in Figure 1. The associated colorings $\phi_{g_{1}}$ and $\phi_{g_{2}}$ are both periodic.

The periodic coloring of the edge-indexed graph of a graph of groups $\Gamma \backslash T$ for $z \mapsto 3 z$ and $z \mapsto z+(1 / 2)$ is as follows:

$$
g_{1}: z \mapsto 3 z, \stackrel{1}{\underset{a}{1}} \quad \stackrel{3}{b}
$$

Here, vertices $a$ and $b$ represent the ideal triangle partitioned as
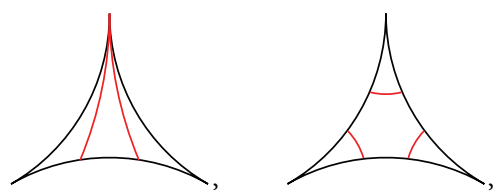

respectively. On the other hand,

$$
g_{2}: z \mapsto z+\frac{1}{2}, \quad \underset{1}{1} \frac{1}{b} \quad \stackrel{1}{\bullet}
$$

In this graph, vertices $a$ and $b$ represent the ideal triangle partitioned as
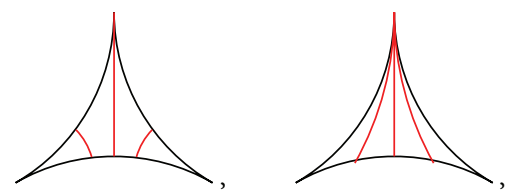

respectively (see Figure 1).

Remark also that an elliptic element $z \mapsto(2 z-1) /(z+1)$ has a periodic coloring identical to that of $z \mapsto 3 z$.

\section{Eventually Periodic Colorings and Their Generalizations}

Now consider an element of $\operatorname{Isom}\left(\mathbb{M}^{2}\right)$ which is not a commensurator of $\Gamma_{r}$. We know that the associated coloring $\phi_{g}$ is not periodic.

Corollary 5. Let $\phi_{g}$ be a coloring associated with an element $g \in \operatorname{Isom}\left(\mathbb{M}^{2}\right)$ which is not a commensurator of $\Gamma_{r}$. Then its associated coloring has infinite alphabet.

Proof. Let $\Gamma^{\prime}=\Gamma_{\mathscr{D}} \cap g \Gamma_{\mathscr{D}} g^{-1}$ and $\Gamma=\Gamma_{r} \cap \Gamma^{\prime}$. By Lemma 2, $\phi_{g}(x)=\phi_{g}(y)$ implies that $x, y$ are in the same right coset of $\Gamma$. Therefore the coloring $\phi_{g}$ has a finite alphabet if and only if $\Gamma$ is a finite index subgroup of $\Gamma_{r}$. By Theorem 3, finiteness of the coloring alphabet is equivalent to the fact that $g$ is a commensurator of $\Gamma_{r}$. 


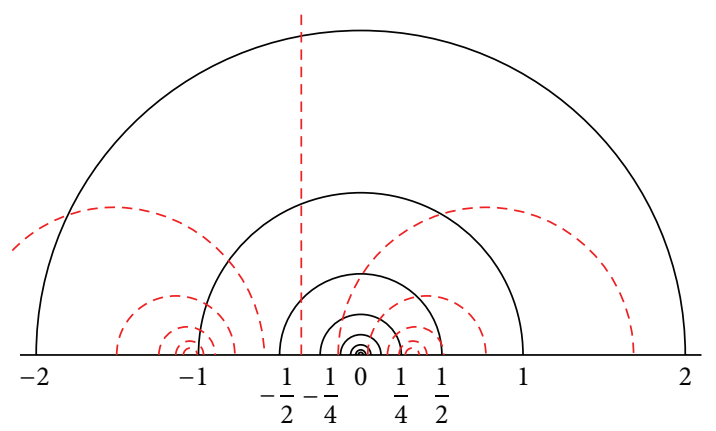

Figure $2: z \mapsto(z-\sqrt{11}) /(\sqrt{10}(z+1))$.

This phenomenon is in contrast to the motivating example of circle rotation explained in the beginning of the last section. In that case, irrational rotations correspond to nonperiodic colorings. However they are defined on a finite set of alphabets, and the corresponding sequences are Sturmian, that is, sequences with subword complexity $p(n)=$ $n+1$. See [9] for Sturmian sequences.

Now let us explain how to obtain colorings of "low complexity" with a finite set of alphabets from hyperbolic tessellations by disregarding some information, as the coloring on a 2-regular tree $\phi_{g}^{\#}$ in (1) for noncommensurable $g$ is Sturmian.

Definition 6. One calls a coloring $\phi$ eventually periodic if there exists a subtree $K$ of finite number of vertices such that $T-K=\cup T_{i}$ is a finite union of subtrees $T_{i}$ such that $\phi$ on each $T_{i}$ can be extended to a periodic coloring on $T$.

In the next examples, let us denote a geodesic in $\mathbb{M}^{2}$ between points $x, y \in \partial \mathbb{T}^{2}$ by $(x, y)$, and let us call the edges in $\mathbb{R}=\partial \mathbb{T}^{2}$ boundary edges.

Example 7. Let $\mathscr{D}$ be the tessellation of $\mathbb{H}^{2}$ with $D$ a generalized ideal polygon whose edges are two geodesics $(-2,2),(-1,1)$ and two boundary edges $[-2,-1],[1,2]$. Let $e_{n}=\left(-2^{n}, 2^{n}\right)$. An element of $\mathscr{D}$ is a generalized ideal polygon which is the region bounded by $e_{n}, e_{n+1}$, for some $n \in \mathbb{Z}$, which we denote by $D_{n}$. The dual graph is a 2 -regular tree $T$, and we can naturally denote the element of $V T$ dual to $D_{n}$ by $n \in \mathbb{Z}$.

In this case, the commensurator of $\Gamma_{r}$ is of the form $z \mapsto$ $a z$ and $z \mapsto a / z$ for $a \in \mathbb{R}$. If $g$ is considered as a map on $\mathbb{M}^{2} \cup \partial \mathbb{M}^{2}$, then $g \in \operatorname{Comm}\left(\Gamma_{r}\right)$ if and only if $g(\{0, \infty\})=$ $\{0, \infty\}$.

Let $g \notin \operatorname{Comm}\left(\Gamma_{r}\right)$ and $\phi_{g}^{0}$ be a coloring given by

$$
\phi_{g}^{0}(m)= \begin{cases}a, & \text { if there is } D \in \mathscr{D} \text { such that } g D \subset D_{m} \\ b, & \text { otherwise. }\end{cases}
$$

If the boundary edge $\left[2^{m}, 2^{m+1}\right]$ or $\left[-2^{m+1},-2^{m}\right]$ contains $g(0)$ (or $g(\infty))$ in its interior, then $D_{m}$ contains $g D_{n} \in g \mathscr{D}$, for sufficiently small (or large, resp.) $n$. This is the case when all vertices of $g D_{n}$ are contained in one boundary edge of $D_{m}$.

Otherwise, we claim that there is no $D \in \mathscr{D}$ such that $g D \subset D_{m}$. Indeed, suppose $g D_{n}$ is contained in $D_{m}$. The only remaining case is when the two boundary edges of $g D_{n}$ are contained in both of the boundary edges of $D_{m}$. Let $\gamma$ be the element of $\Gamma$ sending $D_{m}$ to $D_{n}$. Since $g \gamma$ is an isometry of $\mathbb{M}^{2}$ sending $D_{m}$ into itself and the boundary edges of $g\left(D_{m}\right)$ are contained in both of the boundary edges of $D_{m}$, it sends the geodesic segment $\ell$ of minimal distance between $e_{m}, e_{m+1}$, which is the intersection of the $y$-axis with $D_{m}$, to a geodesic segment of minimal distance between $g \gamma e_{m}, g \gamma e_{m+1}$. Thus the distance between $g \gamma e_{m}, g \gamma e_{m+1}$ is bounded above by the length of $\ell \cap g \gamma D_{m}$, which is strictly less than the length of $\ell$, which is the distance between $e_{m}$ and $e_{m+1}$. This contradicts the fact that $g \gamma$ is an isometry.

Therefore, all vertices except for one or two are colored by $b$, and the remaining one or two vertices whose dual generalized ideal polygon contains $g(0)$ or $g(\infty)$ in its interior are colored by $a$. Hence, by omitting one or two vertices, one obtains a periodic coloring. Thus, $\phi_{g}^{0}$ is an eventually periodic coloring.

In Figure 2, an example of $g: z \mapsto(z-\sqrt{11}) /(\sqrt{10}(z+$ $1))$ is presented. In this case, there are exactly two vertices colored by $a$, that is, $\phi_{g}^{0}(m)=a$ for $m=-2,0$ and $\phi_{g}^{0}(m)=b$ otherwise.

Now consider the Farey tessellation $\mathscr{D}$ and the corresponding group $\Gamma$. The dual graph of $\Gamma$ is a 3-regular tree $T$. Let us provide two examples of colorings given by noncommensurable elements of $\Gamma$ in $\operatorname{Isom}\left(\mathbb{H}^{2}\right)$.

Example 8. Let $g: z \mapsto \alpha z$ with irrational $\alpha$. Then $g \notin$ $\operatorname{Comm}\left(\Gamma_{r}\right)$. Let $\phi_{g}^{1}$ be a coloring given by

$$
\phi_{g}^{1}(x)= \begin{cases}a, & \text { if } \exists D \in \mathscr{D} \text { such that } D_{x} \cap g D \text { contains } \\ & \text { a geodesic line } \\ b, & \text { otherwise }\end{cases}
$$

for $x \in V T$ and $D_{x} \in \mathscr{D}$ corresponding to $x$. A geodesic line is contained in $D_{x} \cap g D$ if only if the two ideal triangles $D_{x}$ and $g D$ have two common vertices. Since the only possible rational vertices of $g D$ are 0 and $\infty, \phi_{g}^{1}(x)=a$ if and only if $D_{x}$ corresponds to ideal triangle of vertices $(0,1, \infty)$ or $(-1,0, \infty)$. Therefore, $\phi_{g}^{1}$ is an eventually periodic coloring, and the coloring of the edge-indexed graph of a graph of groups is as follows:

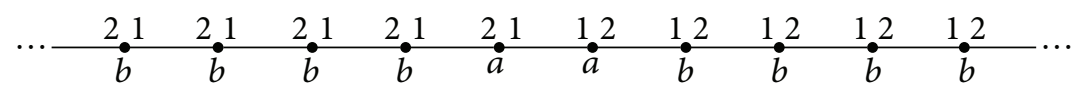

For example, Figure 3 shows the case $g: z \mapsto \sqrt{3} z$. 


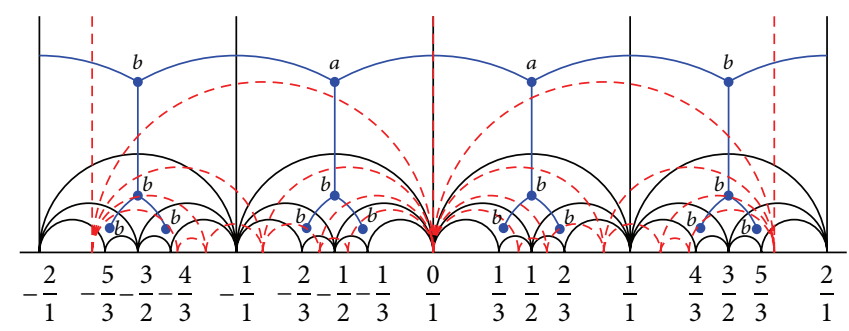

FiguRE 3: $z \mapsto \sqrt{3} z$.

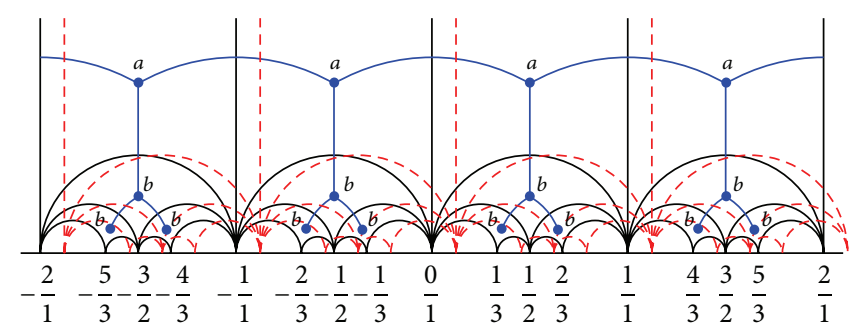

Figure $4: z \mapsto z+\sqrt{17}$.

Example 9. Let $g: z \mapsto z+\beta$ with irrational $\beta$. Then we have $g \notin \operatorname{Comm}\left(\Gamma_{r}\right)$. Let $\phi_{g}^{2}$ be a coloring given by

$$
\phi_{g}^{2}(x)= \begin{cases}a, & \text { if there is } D \in \mathscr{D} \text { such that } D_{x} \cap g D \\ & \text { is not compact, } \\ b, & \text { otherwise }\end{cases}
$$

for $x \in V T$ and $D_{x} \in \mathscr{D}$ corresponding to $x$. If $D_{x} \cap g D$ is not compact, then $D_{x}$ and $g D$ have at least one common vertex. Since all vertices of $g \mathscr{D}$ other than $\infty$ are irrational, $\phi_{g}^{2}(x)=a$ if and only if $D_{x}$ has the vertex of $\infty$, which is the only possible common vertex of $D_{x}$ with $g D \in g \mathscr{D}$. Therefore, $\phi_{g}^{2}$ is a coloring with two colors whose coloring of the edge-indexed graph of a graph of groups is as follows:

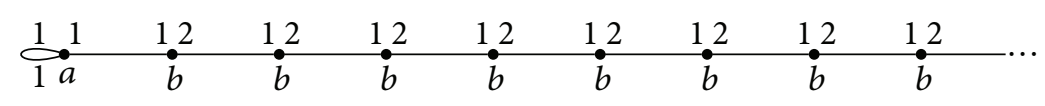

For example, Figure 4 shows the case $g: z \mapsto z+\sqrt{17}$.

We remark that this last example has the number of colored balls up to isometry equal to $n+2$. We believe that the colorings of this type (i.e., with the number of isometry classes of colored balls being $n+2)$ are the ones corresponding to Sturmian sequences. We leave systematic studies about them for future research.

Remark 10. We can generalize the construction in this paper from torsion-free discrete subgroup to any discrete subgroup with one cusp: in this generality, one should consider the minimal subtree containing vertices not in $\partial \mathbb{H}^{2}$, which is again a tree.

\section{Acknowledgments}

The authors would like to thank the anonymous referee for the valuable comments. The first author is supported by NRF 2012R1A1A2004473. The second author is supported by NRF
2012-000-8829, NRF 2012-000-2388, and TJ Park Science Fellowship.

\section{References}

[1] M. Morse and G. A. Hedlund, "Symbolic dynamics II. Sturmian trajectories," American Journal of Mathematics, vol. 62, pp. 1-42, 1940.

[2] G. A. Margulis, Discrete Subgroups of Semisimple Lie Groups, vol. 17 of Ergebnisse der Mathematik und ihrer Grenzgebiete, Springer, Berlin, Germany, 1991.

[3] A. Kubena and A. Thomas, "Density of commensurators for uniform lattices of right-angled buildings," Journal of Group Theory, vol. 15, no. 5, pp. 565-611, 2012.

[4] N. Avni, S. Lim, and E. Nevo, "On commensurator growth," Israel Journal of Mathematics, vol. 188, pp. 259-279, 2012.

[5] V. Platonov and A. Rapinchuk, Algebraic Groups and Number Theory, vol. 139 of Pure and Applied Mathematics, Academic Press, Boston, Mass, USA, 1994, translated from the 1991 Russian original by Rachel Rowen.

[6] H. Bass and R. Kulkarni, "Uniform tree lattices," Journal of the American Mathematical Society, vol. 3, no. 4, pp. 843-902, 1990. 
[7] H. Bass and A. Lubotzky, Tree Lattices, vol. 176 of Progress in Mathematics, Birkhäuser, Boston, Mass, USA, 2001.

[8] J.-P. Serre, Trees, Springer, Berlin, Germany, 1980.

[9] N. P. Fogg, Substitutions in Dynamics, Arithmetics and Combinatorics, vol. 1794 of Lecture Notes in Mathematics, Springer, Berlin, Germany, 2002. 


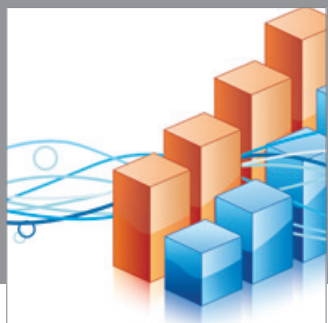

Advances in

Operations Research

mansans

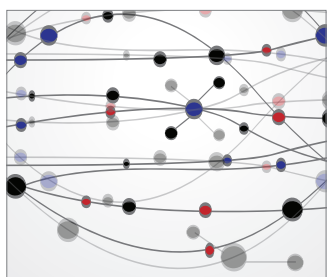

The Scientific World Journal
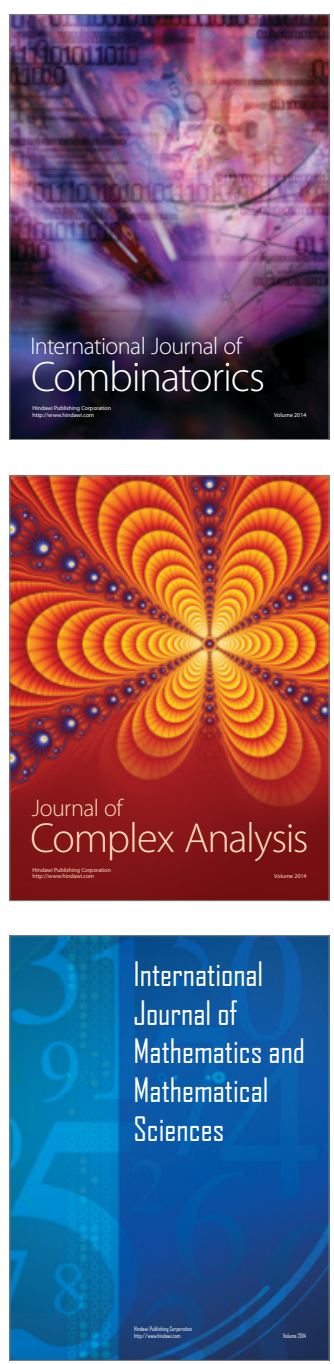
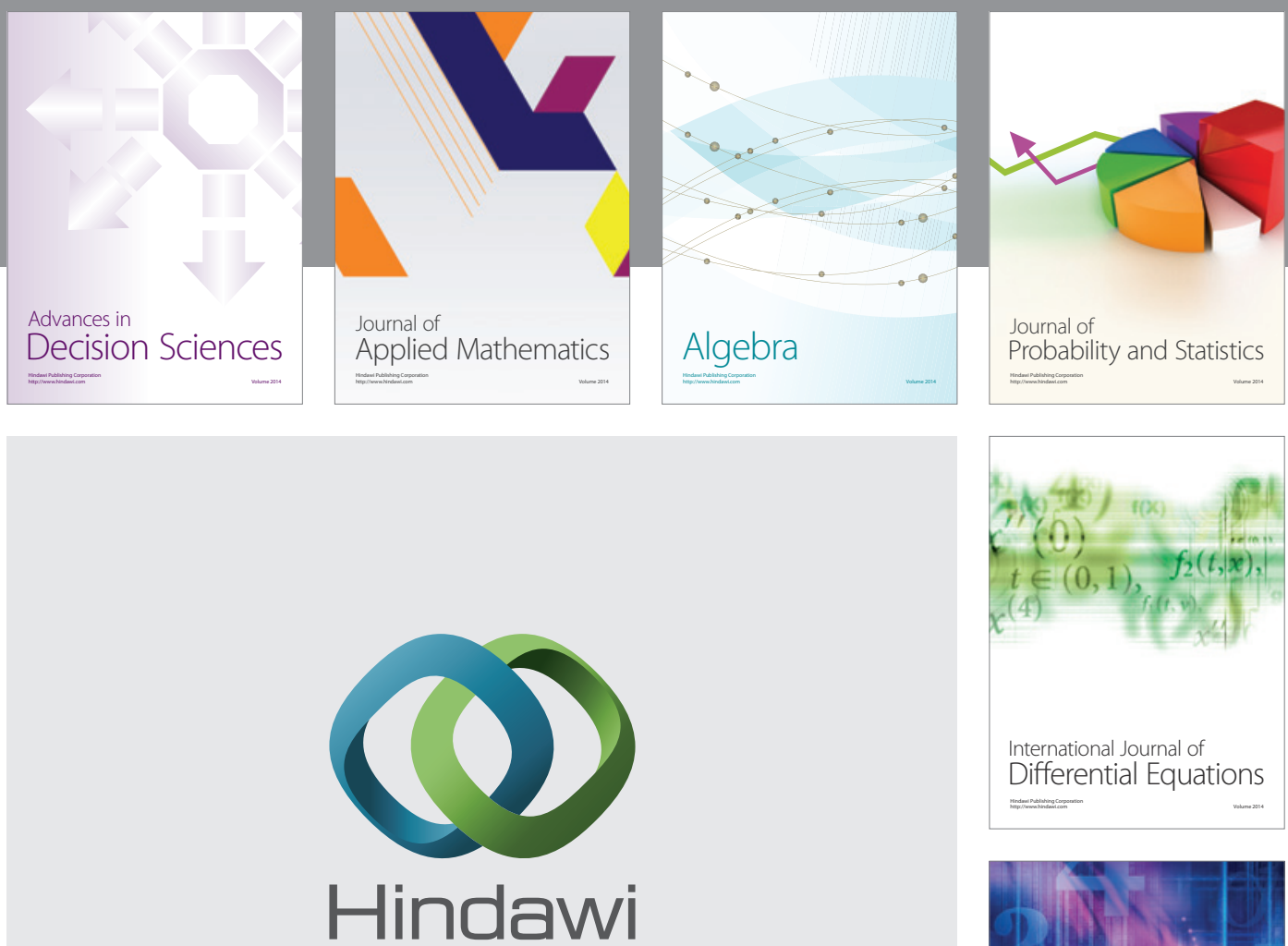

Submit your manuscripts at http://www.hindawi.com
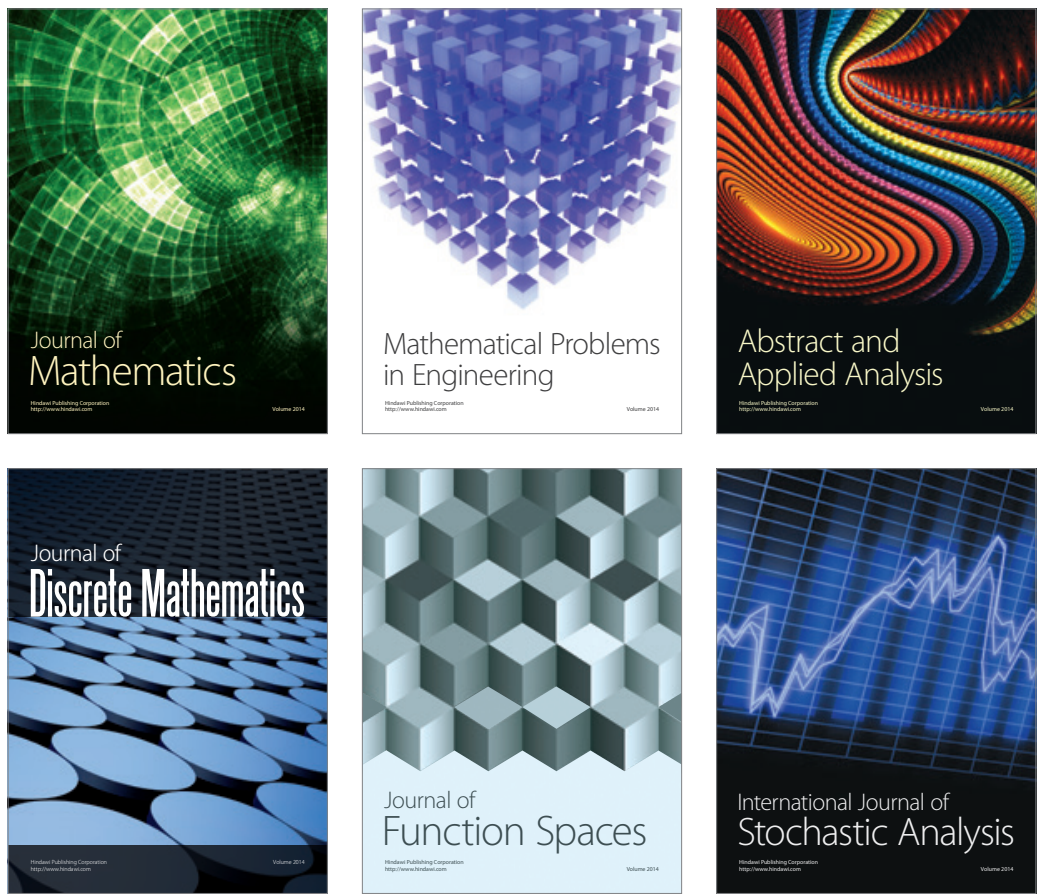

Journal of

Function Spaces

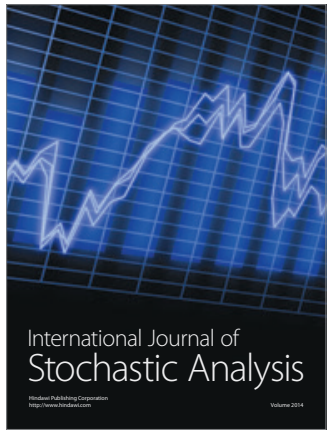

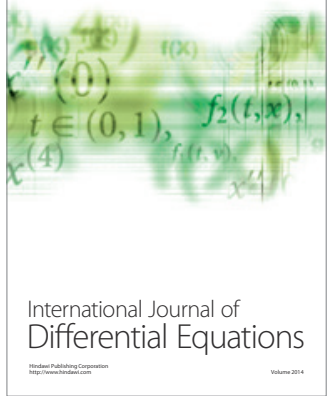
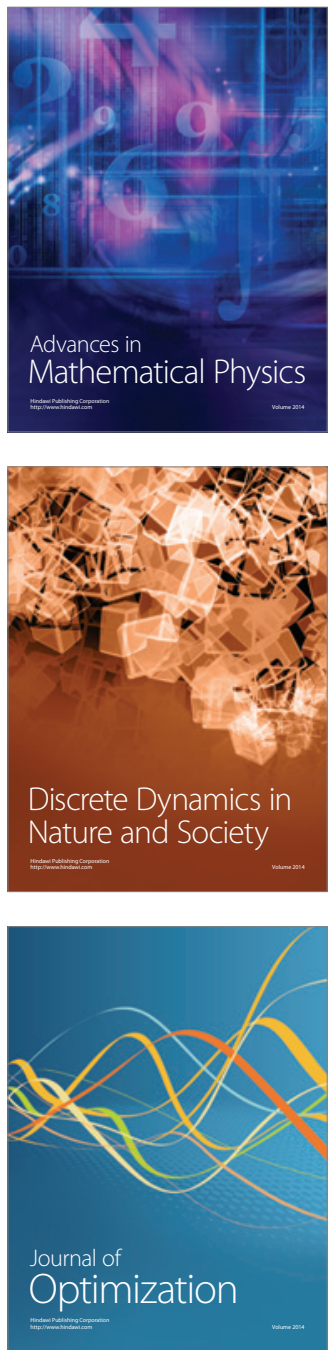